\title{
Möglichkeiten und Leistungsfähigkeit von Finanz- intermediären bei der Finanzierung von KMU
}

\author{
Lothar Brunsch, Martin Grabow
}

In den letzten Jahren ist eine umfangreiche Literatur zu Finanzierungsproblemen von Klein- und mittelständischen Unternehmen (KMU) entstanden, wobei in der Theorie und Praxis sehr viel und zum Teil kontrovers gestritten wird. Mit dem vorliegenden Beitrag wird ausgehend von den Gründen der Auseinandersetzung mit betrieblichen Finanzierungsproblemen der KMU über mögliche theoretische Ausgangsprämissen und Instrumente der Finanzierung die Beteiligungsfinanzierung, insbesondere die der externen Beteiligungsfinanzierung in Form von Finanzintermediären überblicksartig dargestellt.

\section{KMU - Finanzierungsprobleme}

Die Auseinandersetzung mit den betrieblichen Finanzierungsproblemen der KMU soll hier in einer Vorlesungsreihe als Teil der Betriebswirtschaftslehre unter anderem mit folgenden Gründen geführt werden:

1. Die KMU haben in Deutschland eine ungewöhnlich starke Stellung. Von rund 2,1 Mio. Unternehmen sind nach genannter Definition 99,5\% KMU.' Die Stellung der KMU wird unter anderem in folgenden Aussagen noch verdeutlicht:

- am Gesamtumsatz aller Unternehmen haben diese Betriebe einen Anteil von ca. $45 \%$,

- ca. $44 \%$ aller Bruttoinvestitonen werden von ihnen vorgenommen,

- ca. $65 \%$ aller Erwerbstätigen werden durch die KMU beschäftigt,

- ca. $80 \%$ der Auszubildenden werden durch die KMU beschäftigt,

- ca. $50 \%$ des Bruttoinlandsproduktes wird durch sie erarbeitet.

\begin{tabular}{|l|c|c|c|c|}
\hline \multirow{2}{*}{} & \multicolumn{2}{|c|}{ Umsatz } & \multicolumn{2}{c|}{ Beschäftigte } \\
\cline { 2 - 5 } & Anzahl & v.H. & Anzahl & v.H. \\
\hline $\begin{array}{l}\text { GroBunternehmen } \\
\text { (ab 500 Beschaftigte; } \\
\text { ab 50 Mio. DM Umsatz) } \\
\begin{array}{l}\text { mittelständische } \\
\text { Unternehmen }\end{array}\end{array}$ & $\mathbf{8 . 1 6 2}$ & $0,4 \%$ & 3.358 & $0,2 \%$ \\
\hline Summe & 2.013 .662 & $99,6 \%$ & 2.094 .493 & $99,8 \%$ \\
\hline
\end{tabular}

Tabelle 1. Gruppierung der Unternehmensgrößen (vgl. Statistisches Bundesamt 96, S. $527 \mathrm{ff.}$.)

2. Die Existenzsicherung bzw. Existenzgründung jedes Unternehmens in einer wachstumsorientierten Wirtschaft begründet den wachsenden Kapitalbedarf. Die strukturellen Besonderheiten der KMU liegen im besonderen in der diskontinuierlichen Investitionstätigkeit, was wiederum an mangelnden kontinuier- lichen Finanzierungsmöglichkeiten liegt bzw. mit den Managementstrategien bzw. der Unternehmensphilosophie zu begründen ist.

3. Der kontinuierlich wachsende Konkurrenzdruck stellt zunehmend Anforderungen an die KMU. Eine Folge davon ist die verstärkte Technisierung und Rationalisierung, die umfangreiche Investitionen nach sich zieht, was die Bereitstellung von Kapital, einschließlich Eigenkapital, erfordert. Die Kapitalnachfrage wird neben der stofflich materiellen Frage nach neuen Werkstoffen und Produkten immer stärker zur Schliusselfrage für die Zukunft des Strukturwandels und der gesamtwirtschaftlichen Innovationsdynamik.

4. Die Förderung von unternehmerischen Neugründungen, vor allem die Gründung von Jungen Technologieunternehmen (JTU) und Unternehmen mit innovativen Produkten verlangen besondere Formen der Finanzierung in Abhängigkeit von der Gründungsidee. ${ }^{2}$ Jungen Technologieunternehmen wird eine besondere Bedeutung für den dauerhaften Strukturwandel und die internationale Wettbewerbsfähigkeit in Deutschland zugemessen. Folgende Faktoren tragen dazu bei, daß junge Technologieunternehmen verantwortlich für eine dynamische Wirtschaftsentwicklung in Deutschland sind:

- JTU stärken den Innovationswettbewerb, indem sie mit existierenden Unternehmen in Wettbewerb treten und diese dazu zwingen, ihre Innovationsanstrengungen zu verstärken.

- Sie erhöhen bzw. schaffen erst die Nachfrage nach innovationsunterstiitzenden Dienstleistungen.

- Aufgrund ihrer geringen Fertigungstiefe sind sie ein wichtiger Nachfrager für die regionalen $\mathrm{Zu}$ lieferbetriebe.

- Sie schaffen hochqualifizierte Arbeitsplätze sofern die gesetzten Wachstumserwartungen erfüllt werden können.

- Durch Spin-Off's nutzen sie wirtschaftlich die technologischen Entwicklungsergebnisse von Forschungseinrichtungen.

5. KMU haben im allgemeinen keinen unmittelbaren Zugang zum organisierten Kapitalmarkt, d. h., sie können im Gegensatz zu den Publikumsgesellschaften nicht Aktien, Obligationen und Schuldverschreibungen zur Kapitalbeschaffung nutzen. Ihnen bleiben nur begrenzte Möglichkeiten zur Wachstumsfinanzierung. ${ }^{3}$ Wie evident das Problem der Finanzierung bei KMU ist wird auch dadurch deutlich, daß die auf 
Rekordniveau anwachsenden Insolvenzfälle von KMU im wesentlichen auf Managementfehler und Eigenkapitalschwäche zurückgeführt werden. Obgleich ein zu geringes Eigenkapital fur sich betrachtet nicht als eigentliche Insolvenzursache angesehen werden kann, ist die Eigenkapitalhöhe doch ein klares Symptom für fehlende Anpassungsfähigkeit von Unternehmen im Strukturwandel. ${ }^{4}$ Das heißt, die Bewältigung von externen Herausforderungen wird durch interne Mängel blockiert.

6. Die Relevanz der Problematik wird auch dadurch belegt, daß in den letzten Jahren eine umfangreiche Literatur entstanden ist, die sich hauptsächlich mit den Schwierigkeiten bei der Beschaffung des externen Beteiligungskapitals befaßt. Es überwiegen dabei Arbeiten mit empirisch deskriptivem Charakter; Beziehungen zu finanztheoretischen Ansätzen werden aber selten hergestellt. Mit der hier vorgestellten Vorlesungsreihe sollen einige theoretische und praktische Finanzierungsaspekte von KMU, vom theoretischen Ansatz eines informationsbedingten unvollkommenen Kapitalmarktes dargestellt werden. Im Zusammenhang mit den KMU werden nicht behandelt, die sich erstmals den Weg zum organisierten Kapitalmarkt öffnen wollen, die Kreditfinanzierung und die Kopplung leistungswirtschaftlicher und finanzwirtschaftlicher Transaktionen.

Bei der Abhandlung der betrieblichen Finanzierung der KMU wird von folgenden theoretischen Ansätzen ausgegangen:

- Theoretisch handelt es sich hierbei von der Grundstruktur aus um Probleme positiver Theorien mit Berührungsaspekten zu der deskriptiven Theorie, da die Lösung dazu beitragen soll, der zu beratenden Partei logische Unterstützung bei der Bewältigung von Entscheidungsproblemen zu liefern.

- Bei der theoretischen Fundierung und Erklärung dieser finanzwirtschaftlichen Probleme wird von einer betrieblichen Finanzwirtschaft ausgegangen, welche die Investitions- und Finanzierungstheorie gleichermaßen umfaßt, ergänzt um die Disposition der Finanzmittel (als kurzfristiger Steuerungsaspekt) dem Finanzmanagement.

- Es wird weiterhin die Theory of Finance im Sinne einer Finanzierungstheorie im weitesten Sinne als auch die Investitions- und Finanzierungstheorie im engen Sinne betrachtet, wobei die jeweiligen Theorieaspekte der Investitions- und Finanztheorie eine gemeinsame Problemstruktur aufweisen.

- Darüber hinaus ergibt sich dabei die Notwendigkeit, das Zusammenwirken von Investitions- und Finanzierungsentscheidungen im Zusammenhang mit den Finanzmärkten zu sehen und im Sinne einer entscheidungsorientierten BWL Empfehlungen zur Gestaltung der Finanzbeziehungen zu erarbeiten. (vgl. Abbildungen 1 - 5)

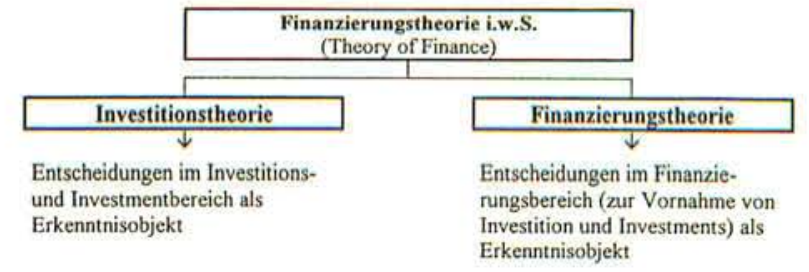

Abb. 1: Aspekte der Finanzierungstheorie i.w.S.

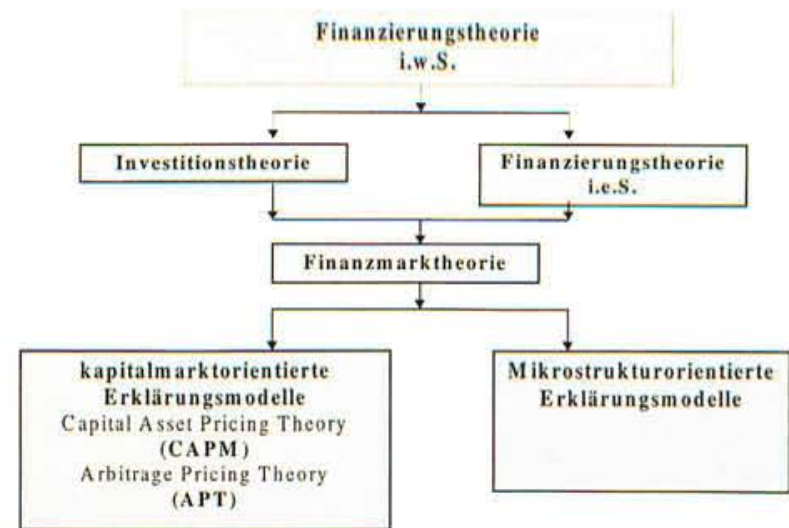

Abb. 2: Finanzmarkttheorie als Klammer zwischen Investitions- und Finanzierungstheorie im engeren Sinne (i.e.S.)

(Unsicherheitsbewältigung)

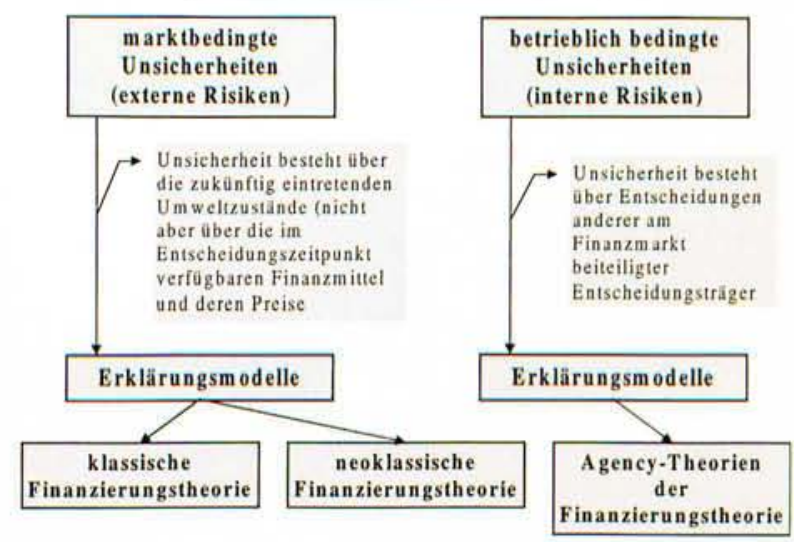

Abb. 3: Klassifizierung von Erklärungsmodellen zur Finanzierungstheorie i.w.S. auf der Basis interner und externer Risiken

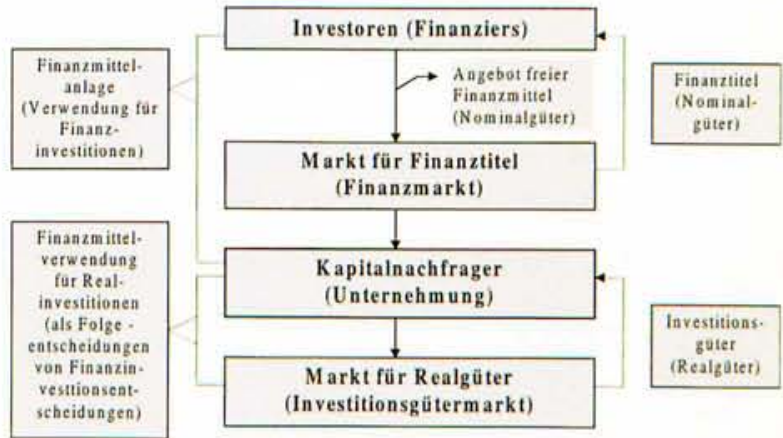

Abb. 4: Beziehungskette zur Integration von Investitions- und Finanztheorie im engeren Sinne (i.e.S.) zu einer Finanztheorie im weiteren Sinne (i.w.S.) 


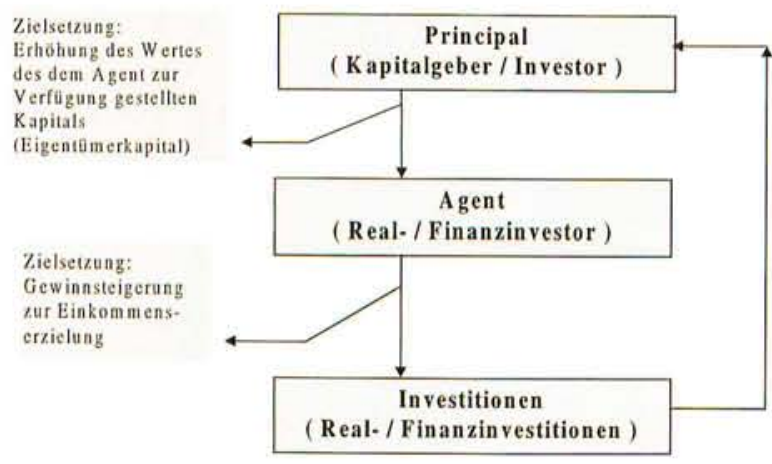

Abb. 5: Verhaltensorientierte Finanzierungstheorie auf der Basis der AgencyTheorie mit entsprechenden Zielsetzungen von Principal und Agent

Hiervon ausgehend werden im weiteren, wie bereits oben erwähnt, theoretische und praktische Aspekte der Kapitalbeschaffung der KMU in Form von

- Finanzierungsinstrumenten,

- Beteiligungsfinanzierungen,

- Beteiligungsgesellschaften aus finanztheoretischer Sicht,

- praktischen Problemen der Beteiligungsgesellschaften,

- Möglichkeiten der Akzeptanz zwischen Kapitalgeber und Kapitalnehmer,

- weitergehenden Problemen

überblicksartig behandelt.

\section{Finanzierungsinstrumente}

Betriebliche Finanzierung (vgl. Abb. 6) kann als die Erhöhung der Ausstattung mit Nominalgütern in dem Sinn aufgefaßt werden, daß sich deren Bestand nach $\mathrm{Ab}$ schluß der Finanzierungsmaßnahmen erhöht. Demzufolge läßt sich Finanzierung einerseits als Prozeß der Versorgung mit Finanzmitteln auffassen; andererseits kann Finanzierung als Ergebnis der Finanzierungsprozesse aufgefaßt werden. Hinsichtlich der Systematisierung der Finanzierungsinstrumente werden im allgemeinen in der Lehre zwei Kriterien herangezogen:

1. Herkunft der aufzubringenden Nominalgüter und

2. Standort der Finanzierungsquelle.

Zunehmend wird der Finanzierungsanlaß (laufende oder ordentliche Finanzierung und Sonderfinanzierung) als ein drittes Kriterium verwendet.

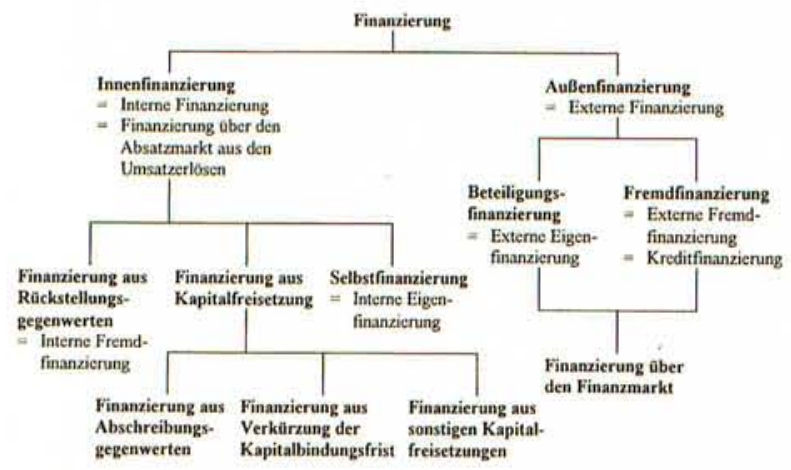

Abb. 6: Finanzierungsalternativen
Ausgehend von der Thematik möchte ich an dieser Stelle nur auf folgende Finanzierungsaspekte eingehen: Untersuchungsaspekte der Deutschen Bank zeigen, daß die Innenfinanzierung in der BRD eine zentrale Rolle bei der Unternehmensfinanzierung spielt. Die Auswertung von ca. 20.000 Jahresabschlüssen von Unternehmen aller Rechtsformen zeigt bis 1991 Tabelle 2:

\begin{tabular}{|l|r|r|r|r|r|r|r|}
\hline & $\mathbf{1 9 8 5}$ & $\mathbf{1 9 8 6}$ & $\mathbf{1 9 8 7}$ & $\mathbf{1 9 8 8}$ & $\mathbf{1 9 8 9}$ & $\mathbf{1 9 9 0}$ & $\mathbf{1 9 9 1}$ \\
\hline Innenfinanzierung & $\mathbf{8 2 , 2}$ & $\mathbf{9 8 , 9}$ & $\mathbf{8 2 , 6}$ & $\mathbf{6 6 , 1}$ & $\mathbf{5 4 , 5}$ & $\mathbf{5 5 , 7}$ & $\mathbf{5 9 , 4}$ \\
Selbstfinanzierung & 4,0 & 11,3 & 10,1 & 5,2 & 0,3 & 3,1 & 2,7 \\
Abschreibungen & 64,0 & $\mathbf{7 3 , 2}$ & 52,4 & 49,9 & 45,0 & 44,7 & 46,8 \\
Rückstellungen & 14,2 & 14,4 & 20,1 & 11,0 & 9,2 & 7,9 & 9,9 \\
& & & & & \\
Außenfinanzierung & $\mathbf{1 7 , 8}$ & $\mathbf{1 , 1}$ & $\mathbf{1 7 , 4}$ & $\mathbf{3 3 , 9}$ & $\mathbf{4 5 , 5}$ & $\mathbf{4 4 , 3}$ & $\mathbf{4 0 , 6}$ \\
Eigenfinanzierung & 3,4 & 3,4 & 2,2 & 2,7 & 5,0 & 6,8 & 4,4 \\
Fremdfinanzierung & 14,4 & $-2,3$ & 15,2 & 31,2 & 40,5 & 37,5 & 36,2 \\
kurzfristiges FK & $\mathbf{7 , 9}$ & $-5,8$ & 16,3 & 23,3 & 34,8 & 30,5 & 30,2 \\
langfristiges FK & 6,5 & 3,5 & $-1,1$ & 7,9 & 5,7 & 7,0 & 6,0 \\
\hline
\end{tabular}

Tabelle 2: Mittelbeschaffung deutscher Unternehmen in $:$

Analysieren wir die Entwicklung der Innenfinanzierung, so ergibt sich Tabelle 3:

\begin{tabular}{|l|r|r|r|r|r|r|r|}
\hline & 1985 & 1986 & 1987 & 1988 & 1989 & 1990 & 1991 \\
\hline Selbstfinanzierung & 3,9 & 14,0 & 13,1 & 7,0 & 0,5 & 4,7 & 4,0 \\
Abschreibungen & 82,9 & 90,8 & 68,0 & 66,9 & 66,7 & 69,1 & 68,4 \\
Rückstellungen & 18,1 & 17,9 & 26,1 & 14,7 & 13,7 & 12,3 & 14,5 \\
\hline E Innenfinanzierung & 104,9 & 122,7 & 107,2 & 88,6 & 80,9 & 86,1 & 86,9 \\
\hline
\end{tabular}

Tabelle 3: Anteil der Innenfinanzierung an den Bruttoinvestitionen in $\%$

Wie die Selbstfinanzierung, unterschieden nach Aktiengesellschaften und aller Rechtsformen aussieht, zeigt die Tabelle 4:

\begin{tabular}{|c|c|c|c|c|}
\hline \multirow{2}{*}{ Jahr } & \multicolumn{2}{|c|}{$\begin{array}{c}\text { Selbstfinanzierung in \% der langfristigen } \\
\text { Mittelbeschaffung }\end{array}$} & \multicolumn{2}{c|}{$\begin{array}{c}\text { Selbsttinanzierung in \% der Brutto- } \\
\text { investitionen in Sachanlagevermogen }\end{array}$} \\
\cline { 2 - 5 } & alle RF & $\mathrm{AG}$ & alle RF & $\mathrm{AG}$ \\
\hline 1975 & - & 7,8 & - & 11,4 \\
1980 & 1,9 & 11,1 & 3,2 & 16,7 \\
1981 & - & 9,2 & - & 13,3 \\
1982 & - & 10,1 & 3,2 & 14,7 \\
1983 & 2,4 & 10,6 & 3,2 & 16,5 \\
1984 & 4,5 & 14,4 & 6,5 & 22,2 \\
1985 & 3,4 & 14,5 & 4,3 & 21,6 \\
1986 & 10,6 & 12,1 & 13,1 & 19,0 \\
\hline
\end{tabular}

Tabelle 4: Selbstfinanzierungsvolumen in \% der Mittelbeschaffung bzw. der Bruttoinvestitionen

Diese Tabellen bestätigen die eingangs gemachten Aussagen, daß die KMU im allgemeinen schlechtere Möglichkeiten haben, die Wachstumsfinanzierung mit Mitteln aus der Innenfinanzierung zu bestreiten, als die Großunternehmen. Die zur Verfügung stehenden Daten lassen nur eine Trendbeschreibung nachvollziehen. Bei der Außenfinanzierung läßt sich anhand der Tabellen die Aussage ableiten, daß große Unternehmen im geringeren Umfang die Fremdfinanzierung nutzen als die KMU. Das deckt sich mit den Aussagen zur Innenfinanzierung.

Aus der hohen Fremdkapitalquote kann sich für die KMU eine ungewollte Abhängigkeit und Einschränkung der Flexibilität im Rahmen der Wachstumsfinanzierung ergeben. Weitere Folgen hoher Fremdkapitalanteile sind:

- Zins- und Tilgungsbelastungen bzw. Liquiditätsbelastungen (unabhängig von der Gewinnsituation können operative Folgen im Hinblick auf Verluste aus Fehlschlägen entstehen).

- Finanzierung über Fremdkapital wird im Regelfall nicht unbegrenzt gewährt. 
- Ein hoher Verschuldungsgrad führt zur Unterlassung risikoreicher Investitionen mit allen Folgen für die zukünftige Wettbewerbsfähigkeit.

Es erhebt sich die Frage, ob diese Finanzierungsprobleme der KMU durch die Einlagen- bzw. Beteiligungsfinanzierung gelöst werden kann?

\section{Beteiligungsfinanzierung 5}

Wie in der Tabelle Mittelbeschaffung dargelegt, liegt die externe Eigenfinanzierung in der Regel zwischen 10 und $15 \%$ im Zeitablauf der externen Finanzierungsmittel. Der Anteil an der genannten Mittelbeschaffung liegt zwischen 2,2 und $6,8 \%$. Hier werden gewisse Abhängigkeiten von der Ertragslage im Vorjahr sichtbar. Die externe Mittelzufuhr als zunächst die schwächste wechselte aber im Zeitablauf ihre Position mit der Selbstfinanzierung. Ein Vergleich von KMU und Großunternehmen bestätigt, daß die Aktiengesellschaften eine merklich höhere Eigenkapitalerhöhung und -ausstattung haben (vgl. Tab. 5). Der Anteil aller Rechtsformen wird noch überzeichnet, da bei Nichtkapitalgesellschaften der Anteil des nicht entnommenen Gewinns noch enthalten ist.

\begin{tabular}{|l|r|r|r|r|r|r|r|r|}
\hline \multicolumn{1}{|c|}{ Jahr } & 1975 & \multicolumn{1}{c|}{1980} & 1981 & 1982 & 1983 & 1984 & 1985 & 1986 \\
\hline alle RF & 7,1 & 2,8 & 2,5 & 1,8 & 4,2 & 5,6 & 3,7 & 3,2 \\
AG & 11,2 & 9,8 & 6,5 & 7,7 & 6,7 & 6,2 & 12,0 & 15,4 \\
\hline
\end{tabular}

Tabelle 5: Eigenkapitalzuführung in \% der langfristigen Mittelbeschaffung

Worin liegen die Ursachen für den geringen Anteil des Eigenkapitals der KMU an der gesamten Mittelbeschaffung?

1. Eine allgemeine Begründung, die auch von der Literatur bestätigt wird, liegt darin, daß die Unternehmen in Deutschland unabhängig von der Rechtsform die Innenfinanzierung vor jeder Form der Außenfinanzierung präferieren.

2. Das unreflektierte Unabhängigkeitsbestreben des Mittelständlers ist selbst ein weiteres Hindernis für die Kapitaldeckung mittels Außenfinanzierung als Beteiligungsfinanzierung.

3. Der sehr geringe externe Eingenfinanzierungsanteil bei den KMU liegt in den beschränkten Möglichkeiten der Beteiligungsfinanzierung selbst, die sowohl von den Kapitalanbietern als auch von der Kapitalnachfragerseite hervorgerufen werden.

4. Durch Investitionsschübe, die vor allem fremdfinanziert werden, verändert sich die Kapitalstruktur zu Ungunsten des Eigenkapitals.

5. Das zentrale Problem bei nicht emissionsfähigen Unternehmen besteht im Auffinden neuer Gesellschafter, da es für derartige Beteiligung keinen Markt gibt. Erschwerend kommt hinzu, daß diese Anteile nicht fungibel sind: sie können also nicht kurzfristig rentabel veräußert werden, hierbei treten weiterhin nicht geringe Transaktionskosten auf und das führt zum Underpricing-Phänomen. Schmidt ${ }^{6}$ wies auf Abschläge bis zu $20 \%$ hin.

Ursachen hierfür sind zum Teil in den asymmetrisch verteilten Informationen zu sehen, denn es gilt in der Literatur die Vermutung, je unsicherer der wahre Wert ist, desto stärker ist das Underpricing. ${ }^{7}$

Die höhere Unsicherheit über die Entwicklung der Unternehmen und die geringen Möglichkeiten der Risikoreduktion schlagen sich folglich in einer höheren Risikoprämie bzw. in einem höheren Preisabschlag nieder.

6. Die Umwandlung auf dem Wege der Gesamtrechtsnachfolge in eine zu gründende AG wäre eine Lösung, um wirtschaftlich an neues Eigenkapital zu kommen. Dagegen sprechen aber folgende Gegebenheiten:

- die hohen Kosten, die dem Unternehmen dabei entstehen,

- die Opportunitätsverluste der veränderten Entscheidungsstruktur,

- die Veränderung der rechtlichen Stellung der Gesellschaft bzw. Gesellschafter,

- die Gefahr der Überforderung usw.

Wegen dieser genannten Beteiligungsfinanzierungsprobleme der KMU werden trotz beträchtlicher Risiken ihre Investitionen zu einem hohen Teil mit Fremdkapital realisiert. Mit der Zunahme des Investitionsdrucks, der durch den hohen Anpassungsbedarf an die sich ändernde Nachfragestrukturen sichtbar wird, werden die Investitionsrisiken und damit die Befriedigung der Ansprüche der Investoren immer unsicherer. Da die KMU überdurchschnittliche Verschuldungsgrade aufweisen, ist die Realisation risikoreicher, aber notwendiger Investitionen, die die notwendigen Wachstumschancen gewährleisten, nur über zusätzliche Anforderungen an das Eigenkapital finanzierbar. Eigenkapital steht auch für die Kraft der Unternehmen, neue Investitionen und Investitionsrisiken zu übernehmen. Aber die bereits gemachten Ausführungen verdeutlichen, daß die KMU erheblichen Problemen bei der Aufnahme von Eigenkapital gegenüberstehen, zum einen durch ihre nicht gegebene Börsenfähigkeit und zum anderen durch ihre ungünstige Finanzierungssituation. Der zweite Aspekt verschärft den Discounteffekt bei nicht handelbaren Anteilen.

Welche Möglichkeiten zur Verbesserung der Eigenkapitalausstattung gibt es für die KMU?

Zum einen wäre die Genußscheinfinanzierung und zum anderen die Finanzierung über das Beteiligungskapital möglich. ${ }^{8}$

Traditionell erfolgt die Finanzierung von Unternehmen durch den direkten Kontakt zwischen den Kapitalgebern und Kapitalnehmern auf dem Kapitalmarkt. Seit geraumer Zeit gibt es auf dem Kapitalmarkt die Möglichkeit für KMU, Kapital von Beteiligungsgesellschaften zu erwerben.

Die Finanzierung über Beteiligungsgesellschaften 
könnte die Probleme der Beteiligungsfinanzierung der KMU beseitigen bzw. mildern. Die Praxis zeigt aber, daß seit der Gründung von Beteiligungsgesellschaften in der BRD Anfang der 60er Jahre bis heute der erwünschte Effekt nicht eingetreten ist. Auch mit dem Gesetz über die Unternehmensbeteiligungsgesellschaften von 1987 konnten die hohen Erwartungen bis heute nicht erfuillt werden. Eine Übersicht hierzu gibt die Abbildung 7 an.

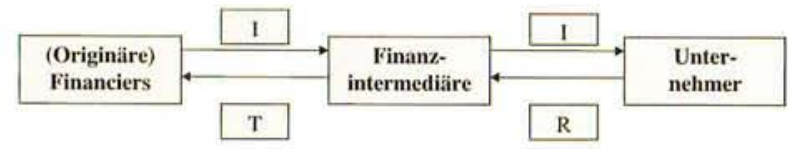

Abb. 7: Finanzierungselemente zwischen den Partnern bei intermediären Finanzierungen von KMU

\section{Beteiligungsgesellschaften als Finanz- intermediäre aus finanzierungstheore- tischer Sicht}

Ein möglicher finanzierungstheoretischer Ansatz ist der neoinstitutionelle der Mikroökonomik, wobei von der Asymmetrie der Informationsverteilung und Handlungsmöglichkeiten ausgegangen wird. Die Wahl dieses theoretischen Ansatzes erlaubt es, im Gegensatz zur Theorie vom unbegrenzten Kapitalmarktzugang, die Besonderheiten der KMU bei der Finanzierung sichtbar zu machen. Das Finanzierungsproblem fuir die KMU stellt sich hierbei in der Art, daß ein Investor Kapital braucht, um eine geplante Investitionsgelegenheit zu nutzen. Damit der Investor das benötigte Kapital bekommt, ist er bereit, einen Teil der zu erwartenden Erträge aus der Investition an den Kapitalgeber abzutreten. Der Investor braucht dabei Kapital für eine längere Frist ohne Gefahr des Abzugs. Im Rahmen der hier zu machenden Ausfuihrungen wird nur auf die Problematik der Beteiligungsfinanzierung eingegangen, wie auf die Agency-Beziehung zwischen Anteilseigner, Kapitaleigentiimer und Manager: ${ }^{9}$

Vergleicht man die Interessen der Kapitalnehmer und der potentiellen Kapitalgeber im allgemeinen miteinander, so ergeben sich folgende, allgemein bekannte Konflikte:

1. Kapitalnehmer wollen eine langfristige Kapitalüberlassung ohne Gefahr des kurzfristigen und überraschenden Abzugs; Kapitalgeber wünschen kurze oder zumindest flexible Anlagezeiten.

2. Kapitalnehmer brauchen zur Durchfuihrung der geplanten Investitionen meist große Kapitalbeträge; Kapitalgeber wollen nur kleine Beträge in ein Unternehmen investieren, um ihr Risiko durch Portefeuillio-Bildung reduzieren zu können.

3. Kapitalnehmer unterliegen ungern der Verpflichtung, zeitlich und betragsmäßig festgelegte Zahlungen leisten zu müssen; Kapitalgeber wünschen verläßliche Zahlungen.
4. Kapitalnehmer sind daran interessiert, riskante Investitionen durchführen zu können; Kapitalgeber sind meist an weniger riskanten Investitionen interessiert.

5. Kapitalnehmer wollen die Möglichkeit haben, Investitionsentscheidungen nach ihren Interessen treffen und ändern zu können und sie wollen dabei viel von dem mit Investition verbundenen Risiko auf Kapitalgeber abwälzen können; Kapitalgeber wollen nicht, daß diese Möglichkeit zu ihrem Nachteil genutzt werden kann. ${ }^{10}$

Bei einer direkten Finanzierung der KMU durch Kapitalgeber stellen sich somit die Interessenkonflikte bezïglich des Finanzierungsvolumens, der Fristen und der Risiken in voller Schärfe, da durch die fehlende Fungibilität der Anteile eine langfristige Bindung unterstellt wird. Des weiteren gibt es Informationskontrollprobleme vor Beginn der Finanzierungsbeziehung und Anreizprobleme nach ihrem Zustandekommen. Daraus erklärt sich, daß ein Kapitalgeber und ein Kapitalnehmer mit kompatiblen Interessen mit geringer Wahrscheinlichkeit zusammenfinden. Diese Wahrscheinlichkeit ist deshalb gering, weil dieser Prozeß zu hohen AgencyKosten sowie zu geringen Renditen führt und für den Kapitalnehmer hohe Kapitalkosten verursacht. Die auftretenden Agency-Probleme können durch institutionelle, organisatorische und vertragliche Vorkehrungen gemildert werden. ${ }^{11}$

Das agency-theoretische Finanzierungsmodell beruht auf dem empirisch zu beobachtenden Sachverhalt, daß vertragsgemäße Beziehungen zwischen den Investoren als Kapitalanbietern und den Unternehmen als Kapitalnachfragern bestehen, wobei auf die in Abbildung 4 wiedergegebene Grundstruktur eines Verhaltensmodells der Finanzierung abgestellt wird.

Eine Möglichkeit, die genannten Probleme bei der Beteiligungsfinanzierung wirtschaftlich zu bewältigen, wird in der Zwischenschaltung einer Kapitalsammelstelle - einer Beteiligungsgesellschaft - gesehen. Die entscheidende Frage hierbei lautet, ob die Zwischenschaltung der Beteiligungsgesellschaft durch ihren institutionellen Apparat eine Senkung der Agency-Kosten ermöglicht, die sich aus Suchkosten, d.h. Informations- und Überwachungskosten, und den finanzierungsbedingten Wertminderungen zusammensetzen. ${ }^{12}$ Die Schaffung von Finanzintermediären kann folgende Vorteile bringen:

- Die Beteiligungsgesellschaft erfuillt durch ausreichende Diversifikation in Form von Portefeuillio-Bildung die Risikoausgleichsaspekte für die Kapitalanleger und sich selbst.

- Durch die Spezialisierung und Verhandlungsmacht der Finanzintermediäre können die Agency-Kosten, die Informations- und Transaktionskosten gesenkt werden.

- Die Kontroll- und Anreizkosten können ebenfalls durch die aktive Rolle und Spezialisierung der zwischengeschalteten Beteiligungsgesellschaft gesenkt werden. 
- Zusätzliche Synergie-Effekte können dadurch erzielt werden, daß die Beteiligungsgesellschaften aktive Managementfunktionen wahrnehmen.

Unter Beteiligungsgesellschaften werden Unternehmen verstanden, deren Geschäftszweck darauf gerichtet ist, die bei ihnen angelegten Gelder im eigenen Namen für gemeinschaftliche Rechnung der Einleger nach dem Grundsatz der Risikostreuung in Beteiligungen an nicht emissionsfähigen mittelständischen Unternehmen anzulegen. ${ }^{13}$ Beteiligungsgesellschaften haben die Stellung eines institutionalisierten Teilhabers, der keine Beherrschung der Partnerfirmen anstrebt, sondern lediglich eine dem Risiko und der Marktsituation angemessene Kapitalrendite. ${ }^{14}$ Häufig tritt auch eine Beratung der jeweiligen Unternehmen hinzu.

$\mathrm{Zu}$ beachten ist, daß durch die Zwischenschaltung von Beteiligungsgesellschaften zusätzliche Transaktionskosten die Kostenvorteile aufsaugen können.

Bei einer Refinanzierung der Beteiligungsgesellschaften über Aktien entstehen wahrscheinlich die geringsten Transaktionskosten, da hier alle Vorteile des organisierten Marktes greifen. Anders ist die Situation bei den Rechtsformen KG und $\mathrm{GmbH}$ der Beteiligungsgesellschaften, denn hier entfällt die Fungibilität und es treten sehr große Losgrößen auf, die einen schnell wachsenden Kapitalbedarf seitens der Kapitalgesellschaften stark einschränken können. Zusätzliche Kosten entstehen durch die Besteuerung der Finanzintermediäre. Die Finanzintermediäre sind steuerlich nicht so gut gestellt, wie der Privatanleger, der sich direkt an einem nichtbörsenfähigen Unternehmen beteiligt.

Diamond versucht mit seiner Theoriekonzeption eine Existenzerklärung von Finanzintermediären. Dabei geht er davon aus, daß der Finanzintermediär nur Bestand hat, wenn er nicht nur die Probleme der originären Finanzierungsbeziehungen gegenüber den kapitalsuchenden Unternehmen besser löst, als es durch die direkte Beziehung zwischen Finanzier und Unternehmen möglich ist, sondern daß die zusätzlichen Kosten der derivativen Finanzierungsbeziehung die Vorteile nicht überkompensieren. An Hand von folgender Konstellation zeigt Diamond, daß dies erfüllt sein kann: Es sind $(\mathrm{M})$ risikoindifferente mittellose Unternehmen mit Investitionsobjekten gegeben, die eine risikolose Verzinsung über den Marktzins erwarten. Die zu erwartenden unsicheren Cash Flow der einzelnen Investitionsobjekte sind voneinander unabhängig und identisch im Intervall $(0, \mathrm{Z})$ verteilt, wobei die hierbei verwendete dichte Funktion allgemein bekannt und nicht veränderbar ist. Die Anfangsauszahlung beträgt I ( I = ganzzahlig). Gleichzeitig gibt es (M) risikoindifferente Finanziers, die jeder mit einer Geldeinheit zur Finanzierung beitragen können. ${ }^{15} \mathrm{Da} \mathrm{I}>1$ ist, sind mehrere Finanziers zur Aufbringung des Kapitals notwendig.

Es wird weiterhin davon ausgegangen, daß der realisierte Cash Flow für die Finanziers nicht kostenlos beobachtbar ist, so daß die Rückzahlungsverpflichtung nicht ohne weiteres auf den Cash Flow abgestellt werden kann. Deshalb muß der Unternehmer durch eine kostenträchtige Kontrolle und durch ein geeignetes Anreizsystem zur Kapitalrückerstattung bewegt werden. Der Abschluß eines Finanzierungsvertrages ohne Anreiz und Kontrolle scheidet aus, denn jeder Unternehmer kann sonst behaupten, der Cash Flow sei Null, d.h. $X=0$, und er würde den tatsächlichen Cash Flow nicht nennen. Zur Kontrolle durch den Finanzier werden Informationen benötigt, deren Beschaffung Informationskosten in Höhe von $\mathrm{C}$ erzeugen. Alternativ bzw. ergänzend kann der Unternehmer auch durch Anreize zur Rüickzahlung bewegt werden. Dabei muß es sich aber um nichtmonetäre Sanktionen handeln, da sie sonst mangels Zahlungsfähigkeit nicht durchgesetzt werden können. Hiervon ausgehend wird der Unternehmer genau diejenige Zahlung an den Finanzier vornehmen, die seinen Nutzen bei dem erreichten Cash Flow und bei einem festgelegten Sanktionsmechanismus maximiert.

In der Regel werden die Finanzintermediäre anstreben, einen vorher von ihnen festzusetzenden Rückzahlungsbetrag $(R)$ mit den KMU zu vereinbaren. Die Ermittlung von $\mathrm{R}$ ist zusammen mit dem Sanktionsmechanismus nach der Theorie von Diamond möglich, die hier nach dem theoretischen Ansatz von Neus/Diamond für Finanzierungen von mittelständischen Unternehmen (KMU) durch Finanzintermediäre ${ }^{16}$ angeführt wird:

Der Finanzintermediär definiert über eine ausgewählte Sanktionshöhe S(T) und durch eine von den Cash Flows (X) der kapitalsuchenden Unternehmen abhängige Ruickzahlung $T$ an seine Finanzgeber eine Wohlfahrtsposition (W). Dabei berücksichtigt er, daß die Finanziers nur Sanktionen akzeptieren, die der expost Nutzensmaximierung entsprechen und die die Rendite in Höhe der Opportunitätskosten sichern. Er hat sodann das folgende Optimierungsproblem zu lösen:

Gesucht wird das max E(W) mit dem Erwartungswert $\mathrm{E}=\mathrm{E}(\mathrm{W})=\int \mathrm{W}(\mathrm{X}) \mathrm{dX}=\int[\mathrm{X}-\mathrm{T}(\mathrm{X})-\mathrm{S}(\mathrm{T})] \mathrm{g}(\mathrm{X}) \mathrm{dX}$ als Zielfunktion unter den Nebenbedingungen

$\mathrm{Z}$

$$
T(X)=\underset{X, S(X), t(X) \leq X}{\operatorname{argmax} E}[X-t(X)-S(t)] \text { und } \int_{0} T(X) g(X) d X \geq i \text { I }
$$

Mit einem Ansatz für die Wohlfahrtsposition W(X), der vor allem die Zahlungsfähigkeit des KMU berüicksichtigt, in der Gestalt

$\mathrm{W}(\mathrm{X})=\mathrm{X}-\mathrm{T}(\mathrm{X})-\mathrm{S}(\mathrm{X})$

ergibt sich folgender Lösungsmechanismus:

$W(X)=\left\{\begin{array}{l}X-R-0=X-R, \text { wenn } X \geq R \\ X-X-(R-X)=X-R, \text { wenn } X<R\end{array}\right.$

also $W(X)=X-R$ bei Beachtung der weiteren Zwischenlösungen von

$R=\min \left(R^{0}\right)$ unter der Bedingung $\left.E\left(\min \left(R^{0}, X\right)\right) \geq i \cdot I\right)$, $\mathrm{T}(\mathrm{X})=\min (\mathrm{R}, \mathrm{X})$

$\mathrm{S}(\mathrm{T})=\mathrm{S}(\mathrm{T}(\mathrm{X}))=\max (0, \mathrm{R}-\mathrm{T})$.

(Theoretischer Ansatz nach Neus/Diamond fuir Finanzierungen von mittelständischen Unternehmen (KMU) durch Finanzintermediäre ${ }^{16}$ ) 


\section{Empirische Aspekte der Beteiligungsge- sellschaften}

Ausgehend von den finanzwirtschaftlichen Gegebenheiten und Wachstumsaussichten der KMU sowie der vorgestellten finanzierungstheoretischen Ansicht wäre zu bemerken, daß Beteiligungsgesellschaften geeignet sind, die Finanzierungsprobleme der nichtemissionsfähigen Unternehmen zu lösen bzw. zu mildern. Das empirische Fondsvolumen der Finanzintermediäre lag 1994 bei ca. 8,8 Milliarden DM, wovon 6,2 Mrd. DM in 2.992 Beteiligungsfällen investiert wurden. Damit verdreifachte sich das Beteiligungskapital in den 90er Jahren. Trotzdem wird dieser Beitrag der Beteiligungsgesellschaften als nicht befriedigend eingeschätzt.

Eine Antwort auf diese relativ geringen Erfolge der Beteiligungsgesellschaften läßt sich unter anderem aus den speziellen Anforderungen der Kapitalnachfrager und Kapitalgeber erkennen. Analysen in beteiligungswilligen Unternehmen zeigen, daß KMU dazu neigen, in erfolgreichen Wirtschaftsphasen unter sich bleiben zu wollen und präferieren die Fremdfinanzierung so lange es die Unternehmensentwicklung erlaubt. Dadurch werden die begrenzten Möglichkeiten der Beteiligungsfinanzierung noch weiter eingeengt. In den Untersuchungen von Wiesenhuber/Spannagel, die rund 500 Unternehmen erfaßte, wurden die Unternehmen auf der Basis von Umsatzwachstum und Umsatzrendite in fünf Klassen eingeteilt. ${ }^{17}$

\begin{tabular}{|c|c|c|c|c|c|}
\hline $\begin{array}{l}\text { Umsatz- } \\
\text { rendite } \\
\text { Umsatz- } \\
\text { wachstum }\end{array}$ & $\begin{array}{l}\text { negativ } \\
(<1 \%)\end{array}$ & $\begin{array}{l}\text { gering } \\
(1 \mathrm{~s}-40)\end{array}$ & $\begin{array}{l}\text { mittel } \\
(5 \%-95)\end{array}$ & $\begin{array}{l}\text { hoch } \\
\left(\geq 1 \frac{1}{3}\right)\end{array}$ & $\begin{array}{r}\text { Umsatz } \\
\text { wachstum }\end{array}$ \\
\hline $\begin{array}{c}\text { stark wachsend } \\
\text { (210\% p.a.) }\end{array}$ & \multicolumn{2}{|c|}{$\begin{array}{l}\text { Umsatzexpansive } \\
\text { Unternelimen }\end{array}$} & \multicolumn{2}{|c|}{ Spitzenunternehmen } & $\begin{array}{c}\text { stark wachsend } \\
\text { ( } 210 \text {; p.a.) }\end{array}$ \\
\hline $\begin{array}{c}\text { wachsend } \\
(16.95 \text { p.a. })\end{array}$ & & \multirow{2}{*}{\multicolumn{2}{|c|}{ Mitläufer }} & & $\begin{array}{c}\text { wachsend } \\
(1 \%, 9 \% \text { p.a. })\end{array}$ \\
\hline $\begin{array}{l}\text { stagnierend } \\
\text { (t }+-03 \text { p.a.) }\end{array}$ & & & & & $\begin{array}{l}\text { stagnierend } \\
(+1-03 \text { p.a.) }\end{array}$ \\
\hline \multirow[t]{2}{*}{$\begin{array}{l}\text { rücklitufig } \\
(<0 \leqslant \text { p.a. })\end{array}$} & \multicolumn{2}{|c|}{ Verlierer } & \multicolumn{2}{|c|}{$\begin{array}{l}\text { Etragsstarke } \\
\text { Unternehmen }\end{array}$} & $\begin{array}{l}\text { rïckliuufig } \\
\text { (<0\% p.a.) }\end{array}$ \\
\hline & $\begin{array}{l}\text { negativ } \\
(<1 \%)\end{array}$ & $\begin{array}{l}\text { gering } \\
(1 \%=49)\end{array}$ & $\begin{array}{l}\text { mittel } \\
(5:-93)\end{array}$ & $\begin{array}{l}\text { hoch } \\
(\geq 15)\end{array}$ & \\
\hline
\end{tabular}

Abb. 8: Klassifizierung der Erfolgstypen ${ }^{18}$

In der Abbildung 8 zeigen sich folgende Ergebnisse:

- Spitzenunternehmen favorisieren die Innenfinanzierung und haben vor allem die geringsten Kredit- und externe Beteiligungsfinanzierungen,

- Verliererunternehmen zeichnen sich durch ein gleichrangiges Verhältnis der Innen- und Kreditfinanzierung aus,

- expansive Unternehmen haben einen höheren Finanzbedarf als alle anderen Unternehmenstypen und die externen Finanzierungsquellen stehen dabei an erster Stelle,

- ca. $30 \%$ der Spitzenunternehmen haben mehr als $50 \%$ Eigenkapitalquote,

- ca. $40 \%$ der umsatzexpansiven Unternehmen und Verlierer sind bis zu $80 \%$ mit Fremdmitteln belastet.

Weitere Ergebnisse der Untersuchungen zeigen, daß Unternehmen, die solche Ziele, wie führende Marktposi- tionen, einer Eigenkapitalrendite über dem Branchendurchschnitt, einen hohen Selbstfinanzierungsgrad und eine hohe Bonität anstreben, sehr häufig zu den Spitzenunternehmen gehören. Verliererunternehmen haben in ihren Unternehmenskonzepten solche Zielstellungen relativ selten. Aber genau diese Unternehmen haben neben den schnell expandierenden Unternehmen einen hohen Bedarf an Beteiligungskapital. Das heißt, Unternehmen suchen häufig erst dann nach Hilfe bzw. neuen Finanzierungsmöglichkeiten, wenn ihre betriebliche Entwicklung schlechte Daten aufweist. Bei den dann einsetzenden Finanzierungsverhandlungen mit eventuellen Finanziers nehmen sie eine schlechtere Ausgangsposition ein, als wenn sie in Zeiten guter wirtschaftlicher Verhältnisse die Verhandlungen strategisch orientiert führen würden.

Die kapitalbeteiligungssuchenden Unternehmen gehören zu denen, die in der Regel ihre bevorzugten Finanzierungsmöglichkeiten ausgeschöpft haben und eine hohe Verschuldungsquote aufweisen. Die zentrale Frage bei der Inanspruchnahme von Beteiligungskapital über die Beteiligungsgesellschaften ist die, ob die Anforderungen und Erwartungen der Beteiligungsgesellschaften mit diesem kapitalsuchenden Klientel übereinstimmen.

Im folgenden wird versucht, aus den möglichen Motiven von Investoren in Beteiligungskapital und den sich daraus ergebenden Formen bzw. Arten der Beteiligungsgesellschaften mögliche Antworten auf diese Fragen zu geben.

Auf dem Beteiligungskapitalmarkt werden drei, vom Grundsatz her unterschiedlich motivierte Investorentypen unterschieden:

1. den finanziell motivierten Investoren, hier dominiert die Erwartung, attraktive Renditen zu erwirtschaften und gleichzeitig das Anlegerrisiko zu minimieren, sie wollen vor allem an den Erträgen partizipieren (Banken und Versicherungen),

2. den strategisch motivierten Investoren, hier dominiert die Erwartung, positive Effekte für das eigene Unternehmen zu finanzieren (Corporate-VentureCapital-Finanzierung),

3. den wirtschaftsfördernden Investoren, hier steht die Förderung der KMU sowie der JTU und innovativen Unternehmen im Vordergrund (Seed Capital Brandenburg GmbH, Frankfurt/Oder).

In der Praxis lassen sich häufig Investoren mit kombinierten Motiven ausmachen.

Bis auf den Typ 3 betreibt der größte Teil der Beteiligungsgesellschaften eine Geschäftspolitik in der Nähe der Kreditwirtschaft.

Diese Forderungen und Bedingungen der Beteiligungsgesellschaften verdeutlichen, daß ihr Kapital nahe dem Fremdkapitalcharakter liegt. Schwierigkeiten beim Auf- 
einandertreffen mit den Kapitalsuchenden entstehen vor allen Dingen dann, wenn die Kapitalbeteiligungsgesellschaften vorrangig mit Risikominderungs- und Selektionsinstrumenten zur Bewertung der kapitalsuchenden Unternehmen arbeiten. ${ }^{19}$ Das heißt, sie erwarten Mindestrenditen, die die Kosten für Fremdkapital übersteigen und Gewinn- und Verlustbeteiligungsmodalitäten, die die Grenzen zur Fremdkapitalfinanzierung verwischen.

Aus diesen drei Investorentypen lassen sich folgende Arten von Beteiligungsgesellschaften definieren:

- Universal-Kapitalbeteiligungsgesellschaften

- Öffentlich geförderte Kapitalbeteiligungsgesellschaften

- Venture-Capital-Gesellschaften

- Unternehmensbeteiligungsgesellschaften

- Seed Capital Gesellschaften

- Sanierungskapitalgesellschaften (ab 1997, Niedersachsen)

Zusammenfassend läßt sich an dieser Stelle feststellen, daß es nicht an kapitalsuchenden Unternehmen fehlt, sondern beide Seiten haben Strategien und Ziele aufgebaut, die schwer mit den Interessen der anderen Seite in Übereinstimmung zu bringen sind. Vereinfacht gesagt, suchen die Beteiligungsgesellschaften kapitalsuchende Unternehmen, die aus Sicht der kapitalsuchenden Unternehmen kein externes Eigenkapital benötigen, und Unternehmen suchen Kapitalbeteiligungen zu Konditionen, die die Beteiligungsgesellschaften häufig nicht erfüllen können oder wollen. Daraus läßt sich erkennen und die praktischen Entwicklungen der letzten Zeit zeigen es, daß auf dem Gebiet des Beteiligungskapitalmarktes weitere Entwicklungen zu erwarten sind. Ziel sollte es sein, für die entsprechenden Kapitalbedarfe der Unternehmen die adäquate Beteiligungsfinanzierungsmöglichkeit zu ermitteln.

Welche Möglichkeiten zur Akzeptanzverbesserung zwischen den Beteiligungsgesellschaften und den kapitalsuchenden KMU gibt es?

1. KMU sollten ihre scheinbare „Herr-im-Haus-Mentalität" überdenken und eine größere Offenheit gegenüber den Beteiligungsgesellschaften zeigen, um die Informationsasymmetrie abzubauen und somit auch das Underpricing zu verringern. ${ }^{20}$

2. Durch Änderungen der geltenden Rechtsvorschriften der Beteiligungsgesellschaften und des Steuerrechts.

- Unter anderem gehören dazu die Veränderungen der Vertragsgestaltung.

- Die Ungleichbehandlung von Eigenkapital und Fremdkapital ist im Steuerrecht weiter abzubauen.

3. Die Beteiligungsgesellschaften sollten eine größere Bereitschaft durch Änderung ihrer Diversifikationspolitik anstreben, um damit Risiken zu mindern und die gegenwärtig favorisierte Selektion zu vermeiden.
4. Bei riskanten Beteiligungen sollten die Beteiligungsgesellschaften zusätzliche Renditen fordern bzw. in unterschiedlichen Entwicklungsphasen modifiziert arbeiten bis hin zur Rückforderung des zur Verfügung gestellten Kapitalbetrages.

5. Die Beteiligungsgesellschaften sollten eine aktivere Rolle in der Geschäftspolitik in den Unternehmen, die ein hohes Risiko fuir sie darstellen, anstreben.

6. Die Beteiligungsgesellschaften sollten sich verstärkt auf den Veräußerungsgewinn durch den Wertezuwachs und nicht auf laufende Ausschuittungen orientieren.

7. Befristete Vertragsabschlüsse sind zu vermeiden, ein Ausstieg aus der Beteiligung sollte von der wirtschaftlichen Entwicklung der KMU abhängig gemacht werden.

8. Die Geschäftsleitung der betreuten KMU sollte am Erfolg des eigenen Unternehmens stärker beteiligt werden.

Die Akzeptanz würde erreicht sein, wenn beide Seiten, die kapitalsuchende und die kapitalgebende, sich als Partner für die Werbung beider wirtschaftlicher Chancen und Interessen annehmen würden.

Bei der Behandlung von Theorie und Praxis ist in diesem Zusammenhang darauf hinzuweisen, daß es zwischen beiden einige generelle unterschiedliche Positionen gibt, z. B. wird in der Theorie das Ratioprinzip verfolgt und in der Praxis das Schutzbedürfnis.

Für die theoretische Arbeit ergeben sich daraus unter anderem folgende Frage- bzw. Problemstellungen:

- Lösungs- bzw. Modelluntersuchungen zur besseren hinreichenden Begründung der Vorteilhaftigkeit von Kapitalbeteiligungsgesellschaften

- Wirkungen einer suboptimalen Kapitalallokation auf die Eigenkapitalausstattung in Unternehmen sowie auf die Entwicklung der Gesamtwohlfahrt

- Ist die Börseneinführung von Unternehmen das geeignete Mittel zur Verbesserung der Eigenkapitalausstattung?

- Erarbeitung von Entscheidungsregeln für die Gestaltung der Verträge zwischen Seed-Capital-Gesellschaften und kapitalsuchenden Unternehmen

- Untersuchungen der Interdependenz zwischen regionaler Strukturpolitik und Beteiligungsgesellschaft und Unternehmen

\section{Fußnoten}

1 Die Ausfuihrungen beziehen sich besonders auf KMU, JTU (Junge Technologieunternehmen) und innovative Unternehmen,

- die nicht emissionsfähig sind (Die Emissionsfähigkeit bezieht sich hierbei auf den potentiellen Börsenzugang eines Unternehmens.), 
- die Umsätze von 50 Mio. DM nicht übersteigen, - die nicht mehr als 500 Arbeitnehmer beschäftigen. Das hervorstechende Merkmal nichtbörsennotierter Unternehmen, so wie es hier behandelt wird, ist, daß es nicht zur Separation von Residualansprïchen einerseits und Entscheidungskompetenzen andererseits kommt. In der Sprache der Property-Rights-Theorie heißt dies, daß alle Ausprägungen von Vertragsrechten in einer Hand vereinigt sind. Diese Unternehmen werden im folgenden, wenn nichts anderes dargelegt wird, nur mit KMU bezeichnet.

2 JTU werden in der Regel von Ingenieuren oder Technikern gegründet, deshalb ist dies besonders ein Problem für Hochschulen, an denen technische Studiengänge existieren. Laut Europäischer Beobachtungsstelle für KMU werden $4 \%$ der heute gegründeten Firmen in diesem Bereich in 10 Jahren für $50 \%$ der Beschäftigung in den überlebenden Unternehmen sorgen. Sie sind die Basis für die zukünftigen wachstumsintensiven Bereiche der Volkswirtschaft.

$3 \mathrm{Zu}$ den hier zu betrachtenden Unternehmen gehören nicht die mit Corporate-Venture-Capital-Finanzierung ausgestatteten Unternehmen. vgl. Schween, K. (1996), S. $13 \mathrm{ff}$.

4 Eine hohe Verschuldung der Unternehmen ist nicht per se ein Zeichen von Ineffizienz, wie es häufig in der Praxis dargestellt wird. vgl. Wiendick (1992), S. 285

5 Auf eine eigene Definition des Eigenkapitals wird an dieser Stelle verzichtet. Vgl. Drukaczyk (1997), Finanzierung, S. $170-177$

6 Schmidt, R.M. u. a., 1988, Underpricing bei deutschen Erstemissionen, 1/84/85, in ZfB, 58 Jg., S. 1193-1203

7 Theoretisch ist ein Underpricing im Durchschnitt erforderlich, um Informationskosten und die Möglichkeit von Verlusten auf seiten des Fianciers auszugleichen. vgl. Rock (1986), S. 276

8 Zur Finanzierung mit Hilfe von Genußscheinen sollen an dieser Stelle keine weiteren Aussagen erfolgen, da ihre Funktionsfähigkeit nur gegeben ist, wenn sie an der Börse gehandelt werden.

9 Vgl. Zu den Interessenskonflikten ... und Vermögensbeziehungen bei Franke G. Hax, Finanzwirtschaft des Unternehmens und Kapitalmarkt, Springer Verlag Heidelberg, 1994, S. $410 \mathrm{ff}$.

10 Vgl. Weingart, S., Zur Leistungsfähigkeit von Finanzintermediären, Peter Lang Verlag 1994, S. 92 und Drukaczyk, Finanzierungstheorie, München, 1980, S. 7

11 Vgl. Aarrow, K.J. (1985), S. 37-51

12 Unter finanzierungsbedingter Wertminderung werden Opportunitätskosten in Form des Nutzenentganges verstanden, der aus dem durch Kontrollrechte beschränkten Handlungsspielraum für Investitions- und Finanzierungsstrategien entsteht.

13 Vgl. Kühr, T., 1989, S. 100-101

14 Vgl. Vallenthin, W., 1976, Sp. 1021; Leopold, G., 1983, S. 170

15 Es gelte $M \geq N \times I$, so daß alle Investitionen finanzierbar sind. Vgl. 4, S. 405-407

$16 \mathrm{Vgl} .13$, S. $109 \mathrm{ff}$.

17 Vgl. Wiesenhuber, N./Spannagel, J. (1988), S. 128 ff.

18 ebenda S. 131

$19 \mathrm{Zu}$ diesen Instrumenten gehören unter anderem die Forderung nach laufender Verzinsung, die Verlustbeteiligung und die Rückkaufmodalitäten. Vgl. auch Wupperfeld, Strategie und Management von Beteiligungsgesellschaften im deutschen Seedkapitalmarkt, Karlsruhe, 1994, S. 68

20 Vgl. Neus, W. (1995); S. $284 \mathrm{ff}$.

\section{Wesentliche verwendete Literatur}

1. Albach, H. (1988): Deregulierung des Aktienrechts, Das Drei-Stufen-Modell; München.

2. Arrow, K.J. (1985): The Economics of Agency, in: Principals and Agents: The Structure of Business; hrsg. von J.W. Pratt/R.I. Zeckhauser, Boston, S. 37-51.

3. Breuer, W. (1993a): Finanzintermeditation im Kapitalmarktgleichgewicht.

4. Diamond, D.W. (1984): Financial Intermediation and Delegated Monitoring, in: Review of Economic Studies; Vol. 51, S. 393-414.

5. Drukaczyk, J. (1993): Theorie und Politik der Finanzierung; 2. Aufl., München.

6. Drukaczyk, J. (1997): Finanzierung; 7. Aufl., Jena.

7. Eilenberger, G. (1997): Betriebliche Finanzwirtschaft; 6. Aufl., München.

8. Franke, G./Hax, H. (1994): Finanzwirtschaft des Unternehmens und Kapitalmarkt; 3. Aufl., Berlin.

9. Hax, H. (1990): Besteuerung, Investitionsanreize und Risikoallokation, zu den theoretischen Grundlagen einer Reform der Unternehmensbesteuerung; in: Aktuelle Fragen der Finanzwirtschaft und Unternehmensbesteuerung; hrsg. v. Dieter Ruickle, 45. Jg., S. 769-779.

10. Kühr, T. (1989): Stand der Venture Capital Finanzierung: Bundesrepublik Deutschland; in: Internationales Venture Capital, hrsg. v. K. Nathasius, Köln, S. 95-115.

11. Jahrmann, F.U. (1996): Finanzierung; 3. Aufl., Herne/Berlin.

12. Laux, H. (1990): Die Irrelevanz erfolgsorientierter Anreizsysteme bei bestimmten Kapitalmarktbedingungen. Der Einperiodenfall; in: Zeitschrift für Betriebswirtschaft, 60. Jg., S. 1341-1358.

13. Neus, W. (1995): Zur Theorie der Finanzierung kleiner Unternehmen; neue betriebswirtschaftliche forschung, Nr. 142; Wiesbaden.

14. Peridon, K./Steiner, M. (1993): Finanzwirtschaft der Unternehmung; 7. Aufl., München.

15. Rock, K. (1986): Why New Issues Are Underpriced; in: Journal of Finanzial Economics; Vol. 15, S. 187 - 212.

16. Schmidt, R.M. u.a. (1988): Underpricing bei deutschen Erstemissionen; in: Zeitschrift für Betriebswirtschaft, 58. Jg., S. 1193-1203.

17. Schween, K. (1996): Corporate Venture Capital; Wiesbaden.

18. Süchting, J. (1997): Finanzmanagement; 6. Aufl., Wiesbaden.

19. Vallenthin, L. (1983): Der Genußschein - ein Instrument für mittelständige Unternehmen zur Eigenkapitalbeschaffung an der Börse; in: ZGR, 12. Jg., S. 445-175.

20. Weingart, S. (1994): Zur Leistungsfähigkeit von Finanzintermediären; Frankfurt/Main.

21. Wiendick, M. (1992): Unternehmensfinanzierung und Kontrolle durch Banken; Deutschland, Japan, USA.

22. Wiesenhuber, N./Spannagel, J. (1988): Situation und Zukunftsperspektiven von Inhaberunternehmen in der BRD; München.

23. Wupperfeld (1994): Strategien und Management von Beteiligungsgesellschaften im deutschen Seedkapitalmarkt; Karlsruhe.

\section{Verfasser}

Prof. Dr. rer. oec. habil. Lothar Brunsch

Dr. rer. nat. Martin Grabow

Technische Fachhochschule Wildau

Fachbereich Betriebswirtschaft/Wirtschaftsinformatik

Tel. (03375) 508-951 